Tropical Journal of Pharmaceutical Research May 2020; 19 (5): 1105-1111

ISSN: $1596-5996$ (print); 1596-9827 (electronic) (C) Pharmacotherapy Group, Faculty of Pharmacy, University of Benin, Benin City, 300001 Nigeria.

\title{
Potential inappropriate prescribing among ambulatory elderly patients in a geriatric centre in southwestern Nigeria: Beers criteria versus STOPP/START criteria
}

\author{
Wuraola Akande-Sholabi ${ }^{1 *}$, Oluwatobi C Ajilore ${ }^{1}$, Segun J Showande ${ }^{1}$, \\ Lawrence A Adebusoye ${ }^{2}$ \\ ${ }^{1}$ Department of Clinical Pharmacy and Pharmacy Administration, Faculty of Pharmacy, University of Ibadan, ${ }^{2}$ Chief Tony \\ Anenih Geriatric Centre, University College Hospital, Ibadan, Nigeria \\ *For correspondence: Email: wuradol@gmail.com
}

Sent for review: 15 January 2020

Revised accepted: 26 April 2020

\begin{abstract}
Purpose: To identify potentially inappropriate prescribing in ambulatory elderly patients and compare the appropriateness of guidelines; Beers' and Screening Tool of Older Person's Prescription (STOPP)/ Screening Tool to Alert Right Treatment (START) criteria to detect potentially inappropriate prescribing among the elderly.

Methods: A retrospective study was conducted using case files of 335 elderly patients aged $\geq 60$ years between 1st January and 31st December 2016, using a data extraction sheet. The 2015 American Geriatrics Society (AGS)-Beers Criteria, and version 2 of the STOPP and START were subsequently used to identify the Potentially Inappropriate Prescribing (PIP) and Potential Prescribing Omissions (PPOs).

Results: Mean age of patients was $69 \pm 0.4$ years (range 60 - 85 years) and 219 (65.4\%) were females. An average of 4.2 medications per patient prescription was found. The Beers criteria identified $26.5 \%$ PIP, while STOPP criteria identified $57.1 \%$ PIP. START detected 29 PPOs in 15 (4.4\%) of the patient's prescription. The most prevalent disease conditions were hypertension 235 (70.1\%) and osteoarthritis 64 (19.3\%). Polypharmacy was significantly associated with PIP in both Beers $(p=0.002)$ and STOPP $(p=0.001)$ criteria.

Conclusion: The prevalence of PIP is high among elderly patients. The STOPP/START criteria identified a higher proportion of PIP among elderly patients compared with Beers criteria. The frequency of PIP should stimulate efforts to curtail potentially inappropriate prescribing and may require the need for advocating for a national criterion to be adopted by health care professionals in Nigeria.
\end{abstract}

Keywords: Potential inappropriate prescribing, Beers' criteria, STOPP/START criteria, Elderly

This is an Open Access article that uses a fund-ing model which does not charge readers or their institutions for access and distributed under the terms of the Creative Commons Attribution License (http://creativecommons.org/licenses/by/4.0) and the Budapest Open Access Initiative (http://www.budapestopenaccessinitiative.org/read), which permit unrestricted use, distribution, and reproduction in any medium, provided the original work is properly credited.

Tropical Journal of Pharmaceutical Research is indexed by Science Citation Index (SciSearch), Scopus, International Pharmaceutical Abstract, Chemical Abstracts, Embase, Index Copernicus, EBSCO, African Index Medicus, JournalSeek, Journal Citation Reports/Science Edition, Directory of Open Access Journals (DOAJ), African Journal Online, Bioline International, Open-J-Gate and Pharmacy Abstracts

\section{INTRODUCTION}

The elderly are the fastest growing population subgroup globally, with almost two-third living in developing countries [1]. According to United Nations projection, the elderly population aged 60 years and above in Nigeria is expected to increase to approximately 26 million from 6.98 
million by 2050 [2], and in Africa, from the 68.7 million to 228.5 million by 2050 [3]. Nigeria is at the lead of this growing age bracket, with approximately $7.0 \%$ of Nigerians in the elderly age group in 2014 [4].

The elderly usually have multiple disease conditions, the management of which frequently leads to polypharmacy. The consequences of polypharmacy are poor health outcomes and hospitalization due to adverse drug reactions [57]. Similarly, polypharmacy is also known to lead to the use of potentially inappropriate medications (PIMs) $[8,9]$. These are medications with greater risk of intolerance related to adverse pharmacodynamics, pharmacokinetics or drugdisease interactions when used in the elderly [10].

Potentially inappropriate medications are consequences of inappropriate prescribing among the elderly. Inappropriate prescribing is a prominent challenge in the management of multiple diseases in the elderly owing to its direct correlation with morbidity, mortality and utilization of health funds globally. To address this problem, the American Geriatrics Society Beers criteria (AGS/Beers) $[10,11]$ and the Screening Tool of Older Persons' Prescription (STOPP)/Screening Tool to Alert Right Treatment (START) [12,13] were developed.

These tools/criteria are commonly used by physicians to target risk management strategies of potential inappropriate prescribing (PIP) among the elderly. Several studies employing the use of both criteria have reported the presence of PIMs among elderly patients in Europe 42.1 - 75.4 \% [14] , in Qatar 38.2 \% [15] , in Brazil 29.9 - $39.6 \%$ [16] in Saudi Arabia 37.5 - $57.6 \%$ [17] and in Nigeria 15.7 - $45.6 \%$ $[18,19]$.

In Nigeria, there are few studies that have investigated PIP or the use of potential inappropriate medications among the elderly. These studies revealed PIP as a common occurrence among them [18-20]. However, some of these studies utilized Beers criteria alone [1820]. None of the studies so far conducted in Nigeria to our knowledge utilized both the AGS/Beers' and STOPP/START criteria. Thus, since there is no criteria outlining the use of medication appropriately or inappropriately among the elderly in the country, this study evaluated the prevalence of PIP and PIMs in the elderly using both the AGS/Beers criteria and STOPP/START with a view to advocating for a criteria that can be adapted for the country.

\section{METHODS}

\section{Study design and study site}

This was a retrospective cross-sectional, hospital-based study carried out at the Chief Tony Anenih Geriatric Centre (CTAGC), University College Hospital (UCH), Ibadan. The centre was recently established and it is the only one currently available in the country. The centre is solely dedicated to the care of the elderly and has about fourteen specialty units. Patients are regularly referred to the centre from other secondary and tertiary health facilities in the country.

\section{Sample size and sampling technique}

Leslie and Kish formula for single proportion using the prevalence of $31.0 \%$ from the study on PIMs use among older Nigerians [19] was used to determine the sample size. Thus, 335 case files of elderly patients at the CTAGC, $\mathrm{UCH}$ were selected. The case files were provided by the Medical Records Department of the centre. Systematic random sampling method was used to enlist every third elderly patients case file until the sample size was complete (sampling interval $\mathrm{k}=\mathrm{NT} / \mathrm{NS}=3.2)$. Where $\mathrm{NT}$ is the sampling frame (1080) and NS = sample size 335.

\section{Data collection}

A data extraction sheet was used to obtain information from the patients' prescription and physician notes in the case files. Data retrieved from individual case files included sociodemographic characteristics of the patients, prescribed medications, number of prescribed medications, diagnosis and laboratory results.

These data were from the most recent prescription and physician notes on the patient. Data collection and entry was carried out by the investigators. Where there were missing or unclear information in the case files, these were verified with the professionals in charge of the unit (physicians, nurses or pharmacists).

Information on the medications use which included the types of medication and PIMs were identified and classified using the American Geriatrics Society 2015 updated Beers criteria by applying the categories; medications to be avoided and used with caution for most older adults and version 2 of STOPP/START criteria to recognize the appropriateness of the drug prescribed (STOPP criteria) and the Potential Prescription Omissions (START criteria). 


\section{Inclusion and exclusion criteria}

Case files of patients aged 60 years and above who attended the CTAGC, University College Hospital, Ibadan between $1^{\text {st }}$ January 2016 and $31^{\text {st }}$ December 2016 were retrieved and used in the study. Sixty years and above was the age adopted by the United Nations and the CTAGC as the definition of older persons. This is also supported by the short life expectancy in Nigeria which is $55 / 56$ years (male/female) ratio according to the United Nations [3]. Case files of patients aged 60 years from other centres in the University College Hospital who did not attend the primary centre for the elderly were excluded from the study. Also, case files of patients without prescriptions, those with incomplete information such as age, sex, morbidity, and laboratory results were excluded from the study.

\section{Data analysis}

Data were sorted, coded and entered into Statistical Package for Social Sciences statistical software version 21.0 (SPSS, IBM Corporation, Armonk, NY, USA) for cleaning and analysis. Descriptive statistics were used to summarise the data. Continuous variables were presented as mean \pm standard deviation (SD), while categorical variables were presented as frequency and percentages. Chi-square statistics was used to determine the association between polypharmacy $(\geq 5$ medications $)$ and multimorbidity (presence of 2 or more diseases), and PIMs based on updated AGS/Beers 2015 and STOPP/START criteria. Where the rule of Chi-square test was violated in the analysis, Fisher Exact test $p$-values were reported for a $2 \mathrm{x}$ 2 contingency table. The value of significance was set at $p<0.05$.

\section{Ethics approval}

Ethics approval for the study was received from the joint University of Ibadan/University College Hospital Institutional Ethical Review Board with approval no. EC/17/0278. The study was carried out by following the principles outlined in the Helsinki Declaration of 1964 [21].

\section{RESULTS}

Three hundred and thirty-five case files were reviewed, with more females 219 (65.4\%) than male. The mean age of the patients was $69 \pm 0.4$ years (range $60-85$ years). The mean number of medications per patient was $4.2 \pm 1.4$ (range 1 10) medications. Polypharmacy described as the use of 5 or more medications per patients was found in $147(43.8 \%)$ patients and multi- morbidity was found in $296(88.4 \%)$ of the studied patients. Hypertension 235 (70.1\%) was the most prevalent disease state found in the elderly followed by osteoarthritis 64 (19.3\%) and diabetes 19 (5.8\%). Table 1 describes the demographic and clinical characteristics of the study population.

Table 1: Demographic and clinical characteristics of the study sample

\begin{tabular}{lc}
\hline Variable & Value n (\%) \\
\hline Gender & $116(34.6)$ \\
Male & $219(65.4)$ \\
Female & $69( \pm 0.4)$ \\
Age (years), mean SD & $70.5 \pm 6.0$ \\
Male & $68.3 \pm 6.0$ \\
Female & $4.2 \pm 1.4$ \\
Mean number of medications & $147(43.8)$ \\
Polypharmacy, n (\%) & $298(88.4)$ \\
Multimorbidity, n (\%) & \\
Disease pattern among the elderly, $\mathrm{n}$ & \\
(\%) & $235(70.1)$ \\
Hypertension & $64(19.3)$ \\
Osteoarthritis & $19(5.8)$ \\
Diabetes & $4(1.3)$ \\
Parkinsonism & $13(3.5)$ \\
Others & \\
\hline
\end{tabular}

Using Beers criteria, 89 patients were on PIMs giving a point prevalence of $26.5 \%$ PIP. Majority of the patients $76(85.4 \%)$ used one PIM, while $13(14.6 \%)$ used 2 PIMs. On the other hand, the STOPP criteria identified 191 patients who were on PIMs and this gave a point prevalence of 57.1 $\%$ PIP. More than half of the patients $118(62.0$ $\%)$ used one PIMs, while 61 (31.9\%) used two PIMs and 12 (6.3 \%) used 3 PIMs.

Higher proportions of patients with multimorbidity used medications found in both Beers 55 (18.4 $\%)$ and STOPP $124(41.7 \%)$ than patients without multimorbidity. However, there was no significant association between multimorbidity and the two criteria. Polypharmacy was statistically significantly associated with PIMs in both the Beers $p=0.002$ and STOPP criteria $p=$ 0.001 (Table 2).

In all, Beers criteria identified 12 PIMs were used by the patients. Non-steroidal anti-inflammatory drugs (NSAIDs) (diclofenac, ketoprofen, naproxen, meloxicam, fenoprofen) were the commonest 267 (70.5 \%) PIMs identified, followed by nitrofurantoin 34 (9.0 \%). The frequency distribution is shown in Table 3.

The most commonly used PIMs detected by STOPP criteria were mostly related to the Cardiovascular (52 \%), Musculoskeletal (26\%) and endocrine systems (17 \%), respectively (Table 4). 
Table 2: Factors associated with potential inappropriate medications based on both Beers and STOPP/START criteria in the elderly

\begin{tabular}{|c|c|c|c|c|c|c|c|}
\hline \multirow{3}{*}{$\begin{array}{l}\text { Associated } \\
\text { factor }\end{array}$} & \multicolumn{7}{|c|}{ Criteria for identification of PIMs } \\
\hline & \multirow[t]{2}{*}{ Group } & \multicolumn{2}{|c|}{ Beers } & \multirow[t]{2}{*}{$P$-value ${ }^{a}$} & \multicolumn{2}{|c|}{ STOPP } & \multirow[t]{2}{*}{$P$-value ${ }^{a}$} \\
\hline & & $\begin{array}{c}\text { Present } \\
n(\%)\end{array}$ & $\begin{array}{c}\text { Absent } \\
n(\%)\end{array}$ & & $\begin{array}{c}\text { Present } \\
n(\%)\end{array}$ & $\begin{array}{c}\text { Absent } \\
n(\%)\end{array}$ & \\
\hline Multimorbidity & $\begin{array}{l}\text { YES } \\
\text { NO }\end{array}$ & $\begin{array}{c}55(18.4) \\
4(11.1)\end{array}$ & $\begin{array}{c}241(81.6) \\
35(88.9)\end{array}$ & $0.347^{b}$ & $\begin{array}{c}124(41.7) \\
13(33.3)\end{array}$ & $\begin{array}{c}173(58.3) \\
25(66.7)\end{array}$ & 0.403 \\
\hline Polypharmacy & $\begin{array}{l}\text { YES } \\
\text { NO }\end{array}$ & $\begin{array}{l}39(26.5) \\
20(10.7)\end{array}$ & $\begin{array}{l}108(73.5) \\
168(89.3)\end{array}$ & $0.002^{*}$ & $\begin{array}{l}78(52.9) \\
59(31.3)\end{array}$ & $\begin{array}{c}69(47.1) \\
129(68.7)\end{array}$ & $0.001^{*}$ \\
\hline
\end{tabular}

${ }^{\mathrm{a} C h i}$-square test, ${ }^{\mathrm{b}}$ Fisher Exact test, ${ }^{*} p<0.05$ was considered significant

Table 3: Prevalence of potential inappropriate medications (Beers criteria)

\begin{tabular}{lcc}
\hline PIMs & N & \% \\
\hline Diclofenac & 154 & 40.9 \\
Ketoprofen & 51 & 13.7 \\
Nitrofurantoin & 34 & 9.0 \\
Naproxen & 26 & 6.8 \\
Meloxicam & 26 & 6.8 \\
Rabeprazole & 26 & 6.8 \\
Amitriptyline & 17 & 4.6 \\
Fenoprofen & 9 & 2.3 \\
Methocarbamol & 9 & 2.3 \\
Metoclopramide & 9 & 2.3 \\
Eszopiclone & 9 & 2.3 \\
Digoxin & 9 & 2.3 \\
Total & 379 & 100.0 \\
\hline PIMs = Potential inappropriate medications
\end{tabular}

Table 4: Top 3 PIMs identified by STOPP

\begin{tabular}{ll}
\hline $\begin{array}{l}\text { Potentially inappropriate } \\
\text { medication (STOPP) criteria) }\end{array}$ & $\mathbf{n ~ ( \% )}$ \\
\hline Cardiovascular system & $99(52.0)$ \\
Furosemide & $45(23.6)$ \\
Aspirin & $30(15.7)$ \\
Musculoskeletal system & $50(26.0)$ \\
Diclofenac & $35(18.2)$ \\
Endocrine system & $32(17.0)$ \\
Glibenclamide & $21(11.2)$ \\
\hline
\end{tabular}

*STOPP - Screening tool of older person's prescription

The START criteria identified 29 PPOs, equivalent to $7.6 \%$ of medications examined. These PPOs happened in 15 (4.4\%) of the patients. More than half of the patients $(57.4 \%)$ had one PPO. The most common potential prescribing omissions were detected in the gastrointestinal tract system medications 13 (45 $\%$ ), associated with the lack of laxative use with continuous opioid use. Omissions in the musculoskeletal system medications were also common $10(35.0 \%)$, and was associated with the lack of bisphosphonates, calcium and vitamin $D$ use. The third most common prescribing omission derived from the cardiovascular system, with lack of statin therapy use. Table 5.
Table 5: Potential prescribing omissions identified using START criteria

\begin{tabular}{lc}
\hline $\begin{array}{l}\text { Potential prescribing omissions } \\
\text { (START criteria) }\end{array}$ & N (\%) \\
\hline $\begin{array}{l}\text { Gastrointestinal tract system } \\
\text { Lack of laxative use with continuous } \\
\text { opioid use }\end{array}$ & $13(45.0)$ \\
$\begin{array}{l}\text { Musculoskeletal system } \\
\text { Bisphosphonates in patients taking }\end{array}$ & $10(34.6)$ \\
$\begin{array}{l}\text { corticosteroids therapy } \\
\text { Calcium and vitamin D supplement in } \\
\text { patients with known osteoporosis }\end{array}$ & $4(14.0)$ \\
$\begin{array}{l}\text { Cardiovascular system } \\
\text { Statin therapy omitted with } \\
\text { documented history of coronary } \\
\text { disease }\end{array}$ & \\
\hline *START = Screening Tool to Alert Right Treatment
\end{tabular}

\section{DISCUSSION}

The prevalence of PIP among elderly population in a geriatric centre in Ibadan, southwestern part of Nigeria, was assessed in this study. Most patients were on multiple medications with an average of 4.2 medications per patient. In this study, about $43.8 \%$ elderly patients were on $>5$ medications, this high rate of polypharmacy could be attributed to high frequency of multimorbidity (presence of two or more diseases) which occurred in about $88.4 \%$ of the studied population. The presence of multimorbidity warrants the needs of more medications to be used to cure or alleviate the symptoms of the various diseases. Comparable studies carried out in other centres in Nigeria and South Africa also stated $>4$ average medications were used per elderly patient [18,19]. Polypharmacy was found to be significantly associated with the use of PIMs in this study, this has also been documented by previous study. Polypharmacy and multi-morbidity are common among the older patients and can predispose patients to adverse drug reactions [22].

This study revealed, that (26.5 and $57.1 \%$ ) patients were prescribed PIMs according to 
Beers and STOPP criteria, respectively. The findings of the PIMs according to Beers criteria in this study were higher when compared with the results of previous studies where the prevalence of PIMs were 18.3 and $19.1 \%$ in Ireland and Taiwan, respectively $[9,23]$. But lower when compared with other similar studies conducted in Nigeria where PIMs prevalence ranged from 30.3 to $35.2 \%$ [19-20]. Different study environments and awareness of available guidelines by physicians for PIMs may account for these variations.

Non-steroidal anti-inflammatory drugs (NSAIDs), nitrofurantoin, rabeprazole and amitriptyline were the drugs prescribed inappropriately in the studied patients. The inappropriate prescription of NSAIDs in this study was not surprising as osteoarthritis was the second most common morbidity found in about a fifth of the respondents. Non-steroidal anti-inflammatory drugs are readily available and are the most affordable analgesics in Nigeria. Previous studies conducted in Spain and Nigeria found benzodiazepines and NSAIDs as the most utilized inappropriate medications [18,19,24]. NSAIDs when used for a long duration without gastroprotective agents, or as first line treatment for the management of pain and in the presence of underlying comorbidities such as congestive heart failure, chronic kidney disease and peptic ulcer in the elderly are classified as PIM [11]. Topical NSAIDs, and patches as alternative to oral have been recommended, however they are more expensive and there is presently no documented report to support its effectiveness in managing musculoskeletal pains such as osteoarthritis in the elderly. This probably has restricted physicians in countries with limited resources like Nigeria to the use of effective medicine for pain among elderly patients, thus the high use of oral NSAIDs in this study.

STOPP criteria in this study identified the prevalence of inappropriate medications as 57.1 $\%$, higher than $29 \%$ reported in United Kingdom [25] and $48 \%$ in Spain [24] respectively. One of the top PIMs identified by STOPP in this study was in the cardiovascular system medications, the use of furosemide a loop diuretic as a first line monotherapy for hypertension and for dependent ankle oedema only with no clinical signs of heart failure are classified as PIMs. Thus, a compression hosiery might be more appropriate for the elderly patients with ankle oedema only. The findings in this study can assist to understand the factors which could be connected with PIMs and provide a guide towards the management of the elderly.
Attention on prevalence of PIMs is essential; moreover, it is important to understand the detailed information that will assist physicians to adopt a control for prescribing pattern implemented for the elderly. The START criteria observed some potential prescription omissions such as the lack of laxative use with continuous opioid use, bisphosphate omitted in patients on corticosteroid therapy, calcium and vitamin D supplement in patients with known osteoporosis and statin therapy omitted with documented history of coronary disease. These omissions could contribute to worsen the health condition of the elderly patients, possibly leading to prescribing cascade, which is an overall effect of polypharmacy. Prescribing cascade is where a physician fails to realize that a new symptom is due to omission of a necessary medication, adverse drug reaction or a side effect, and so another drug is deployed to treat this problem.

\section{Clinical implications}

Our findings indicate that overall prevalence of PIMs using either Beers criteria or STOPP/START was high. The association of PIMs with adverse health outcomes brings forth the need for healthcare providers to reduce the occurrence. Pharmacists could play an important role in improving the management of medication use through a multidisciplinary collaborative approach and the recommendations of deprescribing 'deprescribing is the process of gradual removal of potential inappropriate medications with the goal of reducing unnecessary medications and their related problems'. In addition, the recommendation of frequent medication use review, medication reconciliation and the clinical application of existing criteria such as Beers, STOPP/START or other criteria to target risk management strategies of potential inappropriate prescribing (PIP) among the elderly. All these measures should eventually improve the health quality outcomes of the elderly patients.

\section{Strength and weakness of the study}

One of the drawbacks of this report was that the study was carried out in a single geriatric centre in Nigeria. This might affect the generalizability of the findings to elderly patients across Nigeria. In addition, this study did not apply other categories of the 2015 AGS/BEERS criteria, and this was primarily due to the design of the study, being a retrospective study and all the required information were not documented in the prescription and physician notes available. The version 2 STOPP/START criteria was validated for patients aged 65 years and above and our 
study used patients aged 60 years and above, this was due to the study design which compared the appropriateness of Beers' and STOPP/START criteria, Beers criteria was validated in $>60$ years and our study centre defines elderly as $\geq 60$ years. However, this study's strength involves the use of both Beers and STOPPISTART criteria to identify the frequency of PIMs. These results will offer the literature valuable evidence considering potential inappropriate medications use among the elderly in this environment. The potential prescribing omissions listed in the START criteria could be an invaluable resource in the improvement of elderly patient care.

Consequently, the findings in this study shows the need for advocacy to adopt an existing criterion which will further assist in the reduction of potential inappropriate prescribing. However, the AGS/Beers and STOPP/START criteria limitations remains, these tools were developed for the USA and Europe, and adapting it for a resource limited nation like Nigeria may under-or over-estimate the findings because of the lower number and range of medications available in the country. It is also possible that some of the medications documented on the criteria may not have similar antagonistic effects on diverse population.

\section{CONCLUSION}

Potential inappropriate prescribing prevalence in this study is relatively high, irrespective of the tool used. The STOPP/START criteria identified a higher proportion of PIP compared with Beers criteria among elderly patients. The frequency of PIP should stimulate efforts to curtail potentially inappropriate prescribing and may require the need for advocating for a national criterion to be adopted by health care professionals in Nigeria.

\section{DECLARATIONS}

\section{Acknowledgement}

The authors thank the patients whose data were used for this study.

\section{Conflict of interest}

An article of similar title is held in Research Square preprint server (https://www.researchs quare.com/article/rs-1991/v2) and a formal request has been made to Research Square to have the preprint removed from the server.

\section{Contribution of authors}

We declare that this work was done by the authors named in this article and all liabilities pertaining to claims relating to the content of this article will be borne by the authors. WAS had the original idea, developed study protocol, drafted manuscript, contributed in the data collection and data analysis. OCA, SJS, and LAA developed study protocol, contributed in the data collection and data analysis. All authors contributed to the preparation of the manuscript, read and approved the final version.

\section{Open Access}

This is an Open Access article that uses a funding model which does not charge readers or their institutions for access and distributed under the terms of the Creative Commons Attribution License (http://creativecommons.org/licenses/by/ 4.0) and the Budapest Open Access Initiative (http://www.budapestopenaccessinitiative.org/rea d), which permit unrestricted use, distribution, and reproduction in any medium, provided the original work is properly credited.

\section{REFERENCES}

1. US Census Bureau Age: 2000 - Census 2000 Brief. http://www.census.gov/ prod/2001pubs/c2kbr01-12.pdf. Accessed November 27, 2017.

2. United Nations World Population Ageing, In: Department of Economic and Social Affairs PD. Editor 2015.

3. United Nations, World population ageing highlights (ST/ESA/SERA/397). http://www.un.org/en/developmen t/desa/population/publications/pdf/ageing/WPA2017_Hig hlights.pdf.2017. Accessed 5 June 2019.

4. National Bureau of Statistics, statistical report on women and men in Nigeria. http://www.nigerianstat.gov.ng/ download/49. 2015. Accessed 7 June 2019.

5. Akande-Sholabi $W$, Adebusoye $L A$, and Olowookere $O$ O. Polypharmacy and factors associated with their prevalence among older patients attending a geriatric centre in South-West Nigeria. West African J Pharm 2018; 21(1): 35-45.

6. Borges EP, Morgado M, Macedo AF. Prescribing omissions in elderly patients admitted to a stroke unit: descriptive study using START criteria. Int J Clin Pharm 2012; 34(3): 481-489.

7. Cadmus EO, Adebusoye LA, Olowookere $O O$, Oluwatosin OG, Owoaje ET, Alonge TO. A descriptive study of the morbidity pattern of older persons presenting at a Geriatric Centre in Southwestern Nigeria. Niger J Clin Pract 2017; 20: 873-878.

8. San-Jose' A, Agust'i A, Vidal X, Formiga F, Lopez-Soto $A$, Fernandez-Moyano A. Inappropriate prescribing to older patients admitted to hospital: a comparison of

Trop J Pharm Res, May 2020; 19(5): 1110 
different tools of misprescribing and underprescribing. Eur J Intern Med 2014; 25(8): 710-716.

9. Ryan C, O'Mahony D, Kennedy J, Weedle P, Byrne S. Potentially inappropriate prescribing in an Irish elderly population in primary care. Br J Clin Pharmacol 2009; 68(6): 936-947. doi:10.1111/j.1365-2125.2009.03531.x.

10. Beers MH, Ouslander JG, Rollingher I, Reuben DB, Brooks J, Beck JC. Explicit criteria for determining inappropriate medication use in nursing home residents. UCLA Division of Geriatric Medicine. Arch Intern Med 1991; 151(9): 1825-1832.

11. American Geriatrics Society Beers Criteria Update Expert P. American Geriatrics Society 2015 Updated Beers Criteria for Potentially Inappropriate Medication Use in Older Adults. J Am Geriatr Soc 2015; 63(11): 22272246. doi:10.1111/jgs. 13702.

12. Gallagher P, O'Mahony D. STOPP (Screening Tool of Older Persons' potentially inappropriate Prescriptions): application to acutely ill elderly patients and comparison with Beers' criteria. Age Ageing 2008; 37(6): 673-679. doi:10.1093/ageing/afn197.

13. O'Mahony D, O'Sullivan D, Byrne S, O'Connor MN, Ryan C, Gallagher P. STOPPISTART criteria for potentially inappropriate prescribing in older people: version 2. Age Ageing 2015; 44(2):213-218. doi:10.1093/ageing lafu145.

14. Costa FA, Periquito C, Carneiro MC, Oliveira $P$, Fernandes Al, Cavaco-Silva $P$. Potentially inappropriate medications in a sample of Portuguese nursing home residents: Does the choice of screening tools matter? Int J Clin Pharm 2016; 38: 1103-1111. doi:10.1007/s11096016-0337-y.

15. Alhmoud E, Khalifa S, Bahi A A. Prevalence and predictors of potentially inappropriate medications among home care elderly patients in Qatar. Int J Clin Pharm 2015; 37: 815-821.

16. Fulone I, Lopes LC. Potentially inappropriate prescriptions for elderly people taking antidepressant: comparative tools. BMC Geriatr 2017; 17(1): 278. doi:10.1186/s12877-017-0674-2.
17. Alhawassi TM, Alatawi W, Alwhaibi M. Prevalence of potentially inappropriate medications use among older adults and risk factors using the 2015 American Geriatrics Society Beers criteria. BMC Geriatr 2019; 19: 154. doi.org/10.1186/s12877-019-1168-1

18. Akande-Sholabi W, Adebusoye LA, Olowookere OO. Potentially inappropriate medication use among older patients attending a geriatric centre in south-west Nigeria. Pharm Pract 2018; 16(3): 1235-1242.

19. Saka SA, Oosthuizen F, Nlooto M. Potential inappropriate prescribing and associated factors among older persons in Nigeria and South Africa. Int J Clin Pharm 2019; 41: 207-214.

20. Eze IH, Olowu OA. Prescribing Patterns and Inappropriate Use of Medications in Elderly Outpatients in a Tertiary Hospital in Nigeria. Trop J Pharm Res 2011; 10(1): 19-25.

21. Snežana, Bošnjak. "The declaration of Helsinki: The cornerstone of research ethics". Archive Oncol 2001; 9 (3): 179-184.

22. Frankenthal D, Lerman $Y$, Kalendaryev E. Potentially inappropriate prescribing among older residents in a geriatric hospital in Israel. Int J Clin Pharm 2013; 35(5): 677-682.

23. Lai HY, Hwang SJ, Chen YC, Chen TJ, Lin MH, Chen LK. Prevalence of the prescribing of potentially inappropriate medications at ambulatory care visits by elderly patients covered by the Taiwanese National Health Insurance program. Clin Ther 2009; 31(8): 1859-1870.

24. Ubeda A, Ferrándiz L, Maicas N, Gomez C, Bonet M, Peris JE. Potentially inappropriate prescribing in institutionalised older patients in Spain: the STOPPSTART criteria compared with the Beers criteria. Pharm Pract 2012; 10(2): 83-91.

25. Bradley MC, Motterlini N, Padmanabhan S, Cahir C, Williams $T$, Fahey $T$ et al. Potentially inappropriate prescribing among older people in the United Kingdom. BMC Geriatr 2014; 14: 72: 1-9. 